\title{
PHYSICOCHEMICAL CHARACTERIZATION OF QUERCETIN-LOADED LIPOSOMES PREPARED BY SONICATION FOR FUNCTIONAL FOOD APPLICATION
}

\author{
NUR AMALINA RAMLI, NORA’AINI ALI* AND SOFIAH HAMZAH
}

Faculty of Ocean Engineering Technology and Informatics, Universiti Malaysia Terengganu, 21030 Kuala Nerus, Terengganu, Malaysia.

*Corresponding author: noraaini@umt.edu.my

Submitted final draft: 4 March $2020 \quad$ Accepted: 17 April 2020

http://doi.org/10.46754/jssm.2020.08.002

\begin{abstract}
This research was intended to synthesize liposome as a nanocarrier to encapsulate quercetin, which is prone to degradation and susceptible to low bioavailability upon oral administration. The liposomes were synthesized by thin-film hydration method and followed by probe sonication for downsizing. Soy phosphatidylcholine (SPC) and cholesterol (CHOL) were employed as the composition of the phospholipid bilayer. Results indicated a dependence of sonication amplitude and time in the formation of free liposomes (FL). The average size of quercetin-loaded liposomes (QL) prepared was $346.4 \mathrm{~nm}$ with a narrow polydispersity index $(0.22)$ and a high magnitude of zeta potential $(-49.6 \mathrm{mV})$. These characterizations depict that a homogenous nanovesicle suspension with high stability was successfully synthesized. Quercetin was incorporated into the liposomes with a high encapsulation efficiency of $90.7 \%$ and loading capacity of $9.3 \%$. This viable nanocarrier perhaps will provide ingenious protection for a wider spectrum of active agents in food and biopharmaceutical products.
\end{abstract}

Keywords: Quercetin, liposome, nanocarrier, sonication, encapsulation efficiency.

Abbreviations: [SPC-soy phosphatidylcholine, Chol-cholesterol, FL-free liposomes, QLquercetin-loaded liposomes, MLV-multilamellar vesicles, LUV-large unilamellar vesicles, SUV-small unilamellar vesicles, DLS-dynamic light scattering, PDI-polydispersity index].

\section{Introduction}

There has been a surge of interest in the utilization of natural antioxidants in recent years for promising new leads in functional food products. Nowadays, functional food is rather oriented to the prevention of diet-related diseases. Concurrent with these diseases that are showing worrying trends worldwide, there is a boost in awareness of modern consumers related to the health care and demand on functional food products enriched with antioxidants to promote better health. Dietary flavonoid has gained extensive attention in recent years as a novel medicinal biomolecule. It is aroused by the potential therapeutic properties arising from the antioxidant activities of the phenolic compound (Panche et al., 2016). Additionally, it is stimulated by the diverse health benefits of this phenolic compound which include antiproliferative, chemopreventive, anticancer and anti-inflammatory (David et al., 2016). Quercetin (3,3',4,5,7-pentahydroxyflavone) as in Figure 1 is a naturally occurring flavonoid and the most robust antioxidant among flavonoids (Panche et al., 2016). Quercetin can be found abundantly in a variety of fruits, vegetables and tea (David et al., 2016). It possesses potent antioxidant activity since it can eliminate highly reactive oxygen species, scavenge free radicals and chelate metal ions (Kumar et al., 2015). These effects are mediated by the functional hydroxyl groups in quercetin (Kumar \& Pandey, 2013).

In spite of that, the subject of its bioavailability limits its application. Rapid clearance from the body, poor aqueous solubility, inactive metabolic product, poor absorption and high metabolic rate reflect its characteristic on bioavailability (Nam et al., 2016). In addition, quercetin was also reported to have toxic effects 
<smiles>CC(=O)c1c(O)cc(O)cc1Oc1ccc(O)c(O)c1</smiles>

Figure 1: Chemical structure of quercetin

at high doses when administered orally or intravenously (Rezaei-Sadabady et al., 2016). This problem triggers researchers to investigate for a solution of a non-toxic drug delivery system to reduce the side effects of the drugs. A promising encapsulation system to protect the low bioavailability of quercetin and reduce its toxicity could be the liposome. In the case of liposome, it is non-toxic, and it can be targeted to specific sites in the body for treatment and can be directed away from areas that are sensitive to the toxic nature of the drugs (Kalepu et al., 2013). Many studies have been conducted on liposomes concerned about the toxicity and side effect of drugs in high doses, however, it is proven that via encapsulation, liposomes could reduce the toxicity by delivering low doses of drugs and consequently reduce the exposure of sensitive cells to the toxic drugs (Elmeshad et al., 2014; Karami et al., 2018).

Considerable attention has been given to liposome as the preferred drug carrier because of its amphiphilic nature (He et al., 2018). The liposome is capable of carrying and transporting both hydrophobic and hydrophilic active agents due to the presence of the lipid bilayer and the aqueous core (Dag \& Oztop, 2017). Besides, liposome has led the rate as a drug delivery system as it is biocompatible, biodegradable and able to release the encapsulated substances on demand (Anwekar et al., 2011). The most extensively used method to prepare liposomes is the thin-film hydration method (Bulbake et al., 2017) which forms large and heterogenous MLV that requires a post-treatment in order to produce homogeneous unilamellar vesicles (Bozzuto \& Mollinari, 2015; Laouini et al., 2012).
Methods such as high-pressure homogenization, sonication or extrusion are conducted to downsize the liposomes into nanovesicles, in which sonication is the most widely reported method (Karami et al., 2018; Zidan \& Aldawsari, 2015). Current theories postulate that sonication is able to fragment MLV into LUV and/or SUV by reducing the number of lamellar and downsizing the liposomes (Akbarzadeh et al., 2013; Çağdaş et al., 2014; Silva et al., 2010). The size of liposomes will decrease due to the energy used to break the lipid bilayer into smaller fragments, which subsequently will be enclosed in vesicle structures (Barba et al., 2014). Different studies demonstrated the relationship between the liposome characteristics and sonication condition in terms of amplitude and duration of inputs (Zidan \& Aldawsari, 2015; Silva et al., 2010).

The emphasis in synthesizing liposomes is lying on the ability to produce the right size of vesicle structure with a high encapsulation efficiency of the desired target of the compound. This current work intended to investigate the sonication operating parameters, in terms of amplitude and time of treatment for their influences on the formation of free unloaded liposomes. Hereby, the physicochemical characterization of the liposomes was reported which covered vesicle size, polydispersity index and zeta potential. Five different formulations of quercetin-loaded liposomes were prepared to study the effect of phospholipid to cholesterol ratio and their performance was evaluated where the attention had been given to the encapsulation efficiency and the quercetin loading. Above all, the ultimate goal of this current work was to obtain the best liposome formulation for a high quercetin capture.

\section{Materials and Methods}

\section{Materials}

Soy lecithin (L- $\alpha$-phosphatidylcholine from soybean with $\geq 99 \%$ (TLC) in lyophilized powder was purchased from Sigma-Aldrich (St. Louis, MO, USA). Quercetin (HPLC grade with $\geq 95 \%$ purity), cholesterol (from sheep's 
wool with $\geq 99 \%$ purity), and phosphate buffer saline (in tablet form, $\mathrm{pH}$ 7.4) were also purchased from Sigma-Aldrich (St. Louis, MO, USA). Chloroform and methanol of analytical grade were obtained from Merck (Darmstadt, Germany).

\section{Quercetin-loaded Liposome Preparation}

QL were prepared by the thin-film hydration method with slight modifications (Tefas et al., 2015; Hao et al., 2017). Briefly, the corresponding amounts of phosphatidylcholine, cholesterol and quercetin were dissolved in $10 \mathrm{~mL}$ of chloroform: methanol $(1: 1 \mathrm{v} / \mathrm{v})$. For FL preparation, the composition of phosphatidylcholine and cholesterol was fixed at 8:1 w/w. Meanwhile, the formulations for QL were varied by weight ratio $(8: 0,8: 1,8: 2,6: 1$, $10: 1 \mathrm{w} / \mathrm{w})$. The quercetin was incorporated into the formulation by dissolving in methanol and subsequently mixed with chloroform solution containing phosphatidylcholine and cholesterol. This solution was then transferred to a $250 \mathrm{~mL}$ round bottom flask. The organic solvent was slowly removed by a rotary evaporator under reduced pressure at $45^{\circ} \mathrm{C}$, rotating at $100 \mathrm{rpm}$. Finally, a dry film was formed on the sidewall of the flask. Afterwards, the lipid film was hydrated by adding $10 \mathrm{~mL}$ phosphate buffer saline (PBS) and the liposomal suspension was kept under continuous stirring at $40{ }^{\circ} \mathrm{C}$, above its phase transition temperature (Tc) for 30 minutes.

Subsequently, the liposome suspension was sonicated by a probe sonicator for downsizing (Silva et al., 2010). It was monitored for every 5 minutes increment within 25 minutes. The flask was plunged into an ice water bath to prevent the heating of samples. A pulsed duty cycle was fixed for all treatments with $8 \mathrm{~s}$ on and $2 \mathrm{~s}$ off. Varying amplitudes $(20 \%, 30 \%, 40 \%)$ were tested to investigate the dependence of physicochemical characteristics of liposomes on these sonication parameters. Immediately after preparation, the liposome suspension was centrifuged at 15000 $\mathrm{rpm}$ at $4^{\circ} \mathrm{C}$ for 30 minutes. The supernatant containing the free quercetin in the suspended stage was removed from the pellet of QL. The collected liposomes were washed with $2 \mathrm{~mL}$ PBS, recentrifuged and resuspended to yield 2 $\mathrm{mL}$ of liposome suspension. Then, the mixture was vortexed until homogenization (Elmeshad et al., 2014).

\section{Morphology}

The morphology of liposomes in solid state was examined by scanning electron microscopy (SEM S26000-N Hitachi). The liposome suspension was completely frozen in $-80^{\circ} \mathrm{C}$ overnight before being placed in a freezedryer. The freeze-dried liposome samples were mounted onto adhesive-taped stubs. These samples were sputter-coated with gold film by an automatic auto-coater to prevent any charging up to the surface by the electron beam.

\section{Ultraviolet/Visible Spectroscopy}

UV-Vis absorption spectroscopy was conducted to determine the characteristic peak of quercetin and to verify its presence in liposome suspension. The UV-Vis spectra of QL were obtained by a spectrophotometer (UV-18000 Shimadzu, Kyoto, Japan) in the range of $200 \mathrm{~nm}$ to $800 \mathrm{~nm}$ with $1 \mathrm{~nm}$ intervals. Samples were measured in a rectangular quartz cuvette with 1 $\mathrm{cm}$ path length at $25^{\circ} \mathrm{C}$.

\section{Fourier Transform Infrared Spectroscopy}

The FT-IR spectrum of lyophilized QL was recorded in the range of $4000-400 \mathrm{~cm}^{-1}$ on a FTIR spectrometer (Spectrum 100 Perkin Elmer, Boston, USA). The sample was deposited in spectroscopic grade potassium bromide $(\mathrm{KBr})$ disks. The spectrum was smoothed, and the baseline was corrected using the spectrometer's built-in software.

\section{Particle Size, Polydispersity Index and Zeta Potential Measurements}

Measurements of particle size (Z-average), polydispersity index (PDI) and zeta potential were measured using a dynamic light-scattering (DLS) instrument (Zetasizer ZS, Malvern Instruments, Worcestershire, UK). Multiple 
scattering effect were prevented by diluting the liposome suspensions with distilled water to a concentration of $3.0 \% \mathrm{v} / \mathrm{v}$. The samples were then transferred into an electrophoresis cuvette to measure the zeta potential.

\section{Encapsulation Efficiency and Quercetin Loading}

The encapsulation efficiency and quercetin loading of the liposomes were determined according to the ultracentrifugation method (Elmeshad et al., 2014; Naeem et al., 2016) with slight modifications. The encapsulation efficiency was evaluated by taking supernatant of the centrifuged liposome. The concentration of free un-encapsulated quercetin was measured through a UV-Vis analysis (UV18000 Shimadzu, Kyoto, Japan) at $373 \mathrm{~nm}$. A calibration curve of quercetin in methanol $\left(\mathrm{R}^{2}=0.997\right)$ was previously constructed as a reference. The encapsulation efficiency and loading were measured as a percentage of quercetin entrapped in the liposomes by using the following equations: free quercetin in liposome suspension, and $\mathrm{W}_{\mathrm{np}}$ was the liposome weight.

\section{Statistical Analysis}

All measurements were presented as the mean \pm standard deviation of the results from at least three independent experiments. Statistical analysis was performed by one-way analysis of variance (ANOVA) and $p$-value $<0.05$ was considered statistically significant.

\section{Results and Discussion}

\section{Morphology}

Figure 2 below shows nanosized vesicles of FL and QL using the freeze-drying method. There were no morphological differences between quercetin-loaded and free unloaded liposomes. No excess quercetin particles were observed in the images suggested that most of the unencapsulated quercetin was removed during the process of purification. The liposomes were almost spherical and well distributed with homogeneous size.

Encapsulation efficiency $(\%)=\left(\mathrm{W}_{\text {total }}-\mathrm{W}_{\text {free }}\right) \times 100 / \mathrm{W}_{\text {total }}$

Quercetin loading $(\%)=\left(\mathrm{W}_{\text {total }}-\mathrm{W}_{\text {free }}\right) \times 100 / \mathrm{W}_{\mathrm{np}}$

where $\mathrm{W}_{\text {total }}$ was the total weight of quercetin in liposome suspension; $\mathrm{W}_{\text {free }}$ was the weight of
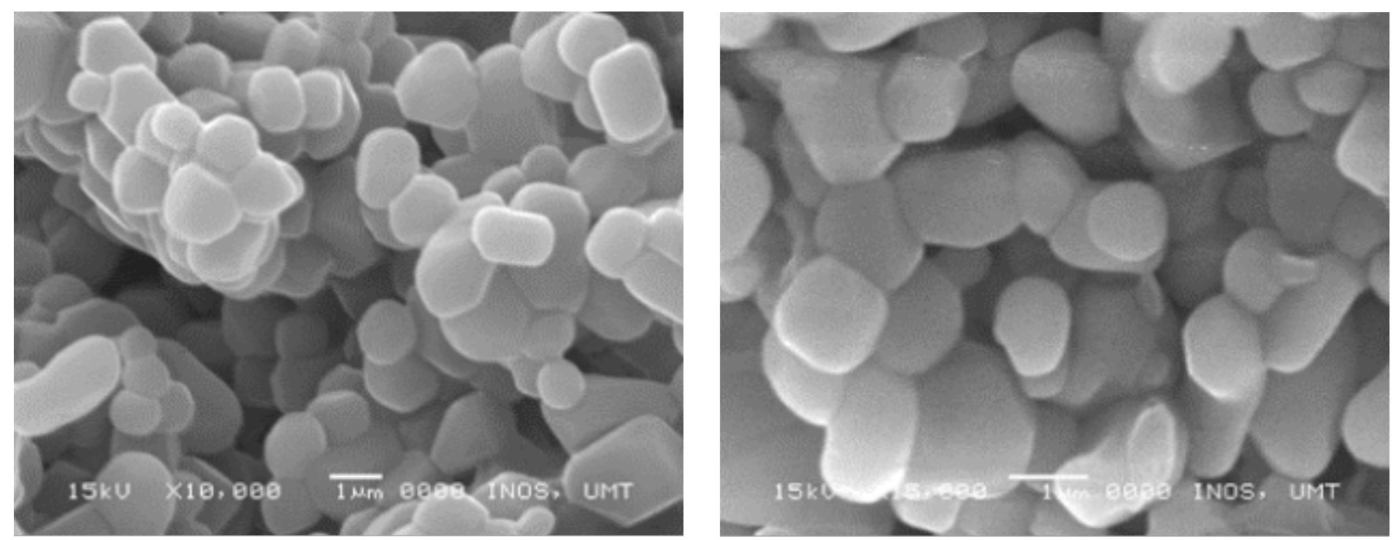

Figure 2: SEM images of (a) FL with magnification of x10 000 (scale $1 \mu \mathrm{m}$ ) and (b) QL with magnification of x15 $000($ scale $1 \mu \mathrm{m})$ 


\section{Ultraviolet-Visible Spectroscopy}

Figure 3 presented ultraviolet-visible (UVVis) spectra of free quercetin, FL and QL. The spectrum of native quercetin showed two absorption bands at $255 \mathrm{~nm}$ and $370 \mathrm{~nm}$, were associated with the conjugations in the B-ring and A-ring of the compound, respectively. The spectrum of free liposomes showed no apparent peak of absorption, similar to those described by Hao et al. (2017). Significant absorption was observed after encapsulation at $255 \mathrm{~nm}$ and $373 \mathrm{~nm}$, confirming the incorporation of quercetin in the liposomes. The peak at $373 \mathrm{~nm}$ increased and exhibited softly Einstein shift after quercetin incorporated in the liposomes. These spectra were in agreement with the results reported previously by Wu et al. (2015) and Hao et al. (2017).

\section{Fourier Transform Infrared Spectroscopy}

The Fourier transform infrared (FTIR) spectra of free quercetin, FL and QL were presented in Figure 4. These FTIR spectra were evaluated to interrogate the viable chemical interaction between quercetin and liposomes. In view of the spectrum of quercetin, the expanded band centered at $3376 \mathrm{~cm}^{-1}$ was prompted by stretching of phenolic $\mathrm{O}-\mathrm{H}$ vibration while around $1747 \mathrm{~cm}^{-1}$ was by the stretching vibrations of carbonyl $(\mathrm{C}=\mathrm{O})$ groups. The band at $1620 \mathrm{~cm}^{-1}$ was ascribed to the aromatic moiety of $\mathrm{C}=\mathrm{C}$ stretching and bands at 1448, 1348 and $812 \mathrm{~cm}^{-1}$ indicated the bending of C-H strains. The characteristic band of $\mathrm{C}-\mathrm{O}$ in the aromatic ring structure appeared at $1228 \mathrm{~cm}^{-1}$ and $\mathrm{C}-\mathrm{OH}$ was obtained at $1184 \mathrm{~cm}^{-1}$.

FL displayed their typical peaks at 2927 $\mathrm{cm}^{-1}$ and $2864 \mathrm{~cm}^{-1}$, which corresponded to the stretching vibration of alkyl groups of phospholipids. The ester groups of phospholipids were shown at $1747 \mathrm{~cm}^{-1}$ indicated the symmetrical $\mathrm{C}=\mathrm{O}$ stretching vibration while the absorption peak at $1080 \mathrm{~cm}^{-1}$ was from C-O stretching bond. The polar head group vibrations were represented by the antisymmetric stretching of the $\mathrm{PO}_{4}$ groups at $1232 \mathrm{~cm}^{-1}$, and the band representing the antisymmetric $\mathrm{N}^{+}-\mathrm{CH}_{3}$ stretching vibrations was at $993 \mathrm{~cm}^{-1}$.

For QL, the peak located at $2927 \mathrm{~cm}^{-1}$ and $2864 \mathrm{~cm}^{-1}\left(\mathrm{CH}_{2}\right)$ was similar to that of FL. The $\mathrm{O}-\mathrm{H}$ stretching band of quercetin at $3376 \mathrm{~cm}^{-1}$ was shifted to $3404 \mathrm{~cm}^{-1}$ which suggested that quercetin interacted with the liposome bilayer by intermolecular hydrogen bonds. The band related to $\mathrm{O}-\mathrm{H}$ stretching from $\mathrm{FL}$ spectrum was at $3418.7 \mathrm{~cm}^{-1}$ with area under the peak of $22.3 \%$ and it was increased to $24.2 \%$ as shown in QL spectrum. This increment was attributed to the hydroxyl group of quercetin incorporated into the phospholipid bilayer of liposomes. The bands for functional groups of quercetin, which depict $\mathrm{C}=\mathrm{O}$ stretching, and $\mathrm{C}-\mathrm{OH}$ also became

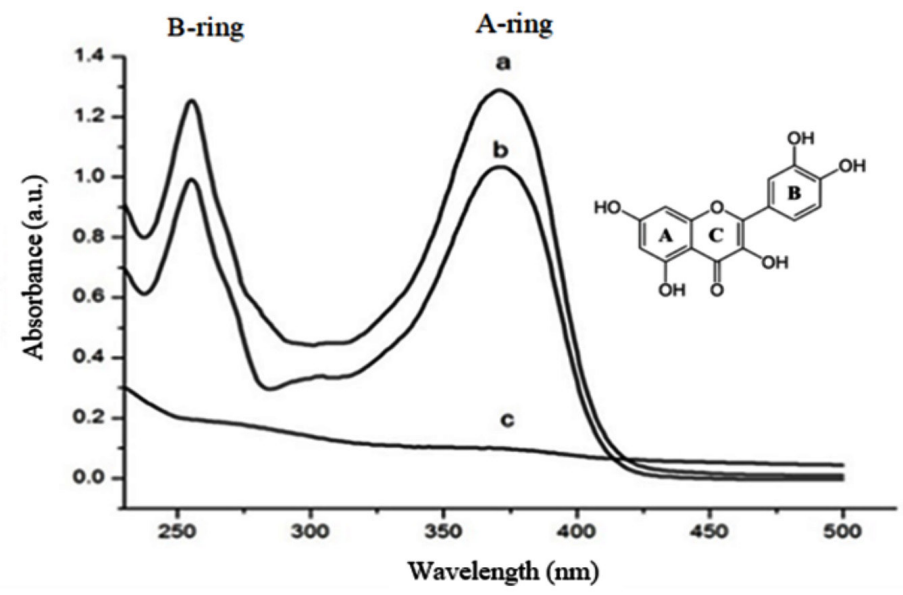

Figure 3: UV-Vis spectra of (a) QL, (b) free quercetin and (c) FL 


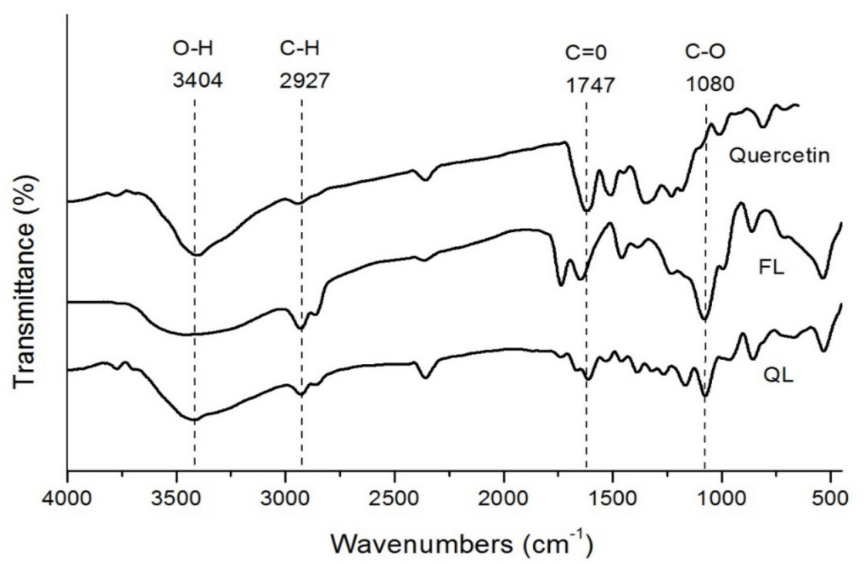

Figure 4: FTIR spectra of (a) free quercetin, (b) FL and (c) QL

broader and nearly disappear in the spectrum of QL. This suggested that quercetin was entrapped within the liposome bilayer, possibly by hydrophobic interactions between the carbonyl group of quercetin and the phospholipid bilayer. This finding was comparable with the study conducted by Hao et al. (2017).

\section{Effect of Sonication Amplitude and Duration on Free Liposomes}

Characterizations of FL concerning particle size are presented in Figure 5, confirming that prepared liposomes were in a nanoparticulate system. Particle size is always recognized as a major concern in synthesizing nanocarrier since large particles larger than $500 \mathrm{~nm}$ were reported to be more likely excreted by the mononuclear phagocyte system (MPS) before reaching the bloodstream (Bozzuto \& Molinari, 2015). In contrast, liposomes with the particle size of 100 $\mathrm{nm}$ to $300 \mathrm{~nm}$ can avoid the possibility of the rapid clearance and have excellent potency to transport the encapsulated materials between and into cell membranes (Li et al., 2016). The smallest average size of free liposomes obtained was $229.3 \mathrm{~nm}$, which was prepared at sonication amplitude of $40 \%$ for 25 minutes. The particle size was inversely related to the sonication parameters as the size was decreasing $(\mathrm{p}>0.05)$ with the increase of time and amplitude. These results were in accordance with the finding by Silva et al. (2010), who also discovered the inverse relationship between these parameters.

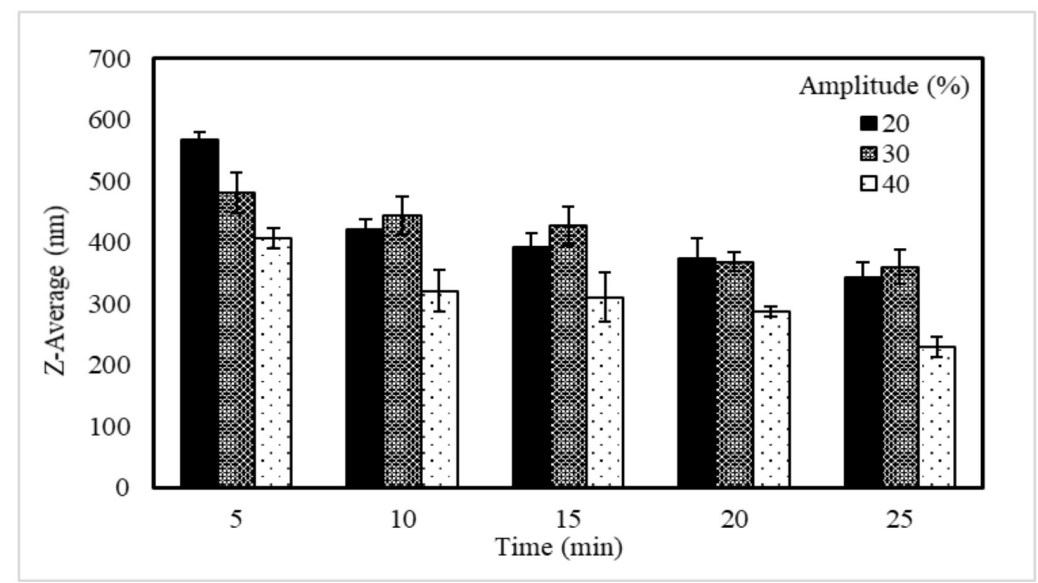

Figure 5: Effect of sonication amplitude and time on particle size of FL 
The sonication was continued for 25 minutes to reduce the possibility of lipid and probe degradation as it might occur due to the high energy and heat are given off during treatment.

The size distribution of liposomes can be represented by the polydispersity index and a smaller value of the index indicates a narrow distribution of the particle size (Zhao \& Temelli, 2017). All the indices of liposomes measured throughout the sonication as presented in Figure 6 were lower than 0.3 . These results suggest that the liposomes were monodispersed. Herein, sonication can be considered as an efficient method to produce a well-dispersed liposome system. There was less significant change on the PDI value of liposomes within the sonication period. Regardless of that, the lowest PDI value after sonication was noted at 0.205 with the amplitude of $40 \%$ after 25 minutes. It was at the same point as the smallest particle size was achieved.

Apart from particle size and polydispersity index, the zeta potential of liposome suspension was also measured according to the effective electric charge on the liposome surface, which presented the fate of the colloidal stability (Honary \& Zahir, 2013). In Figure 7, zeta potential of FL shows an increment $(p<0.05)$ with treatment time regardless of the amplitude used for sonication. This could be the after- effect of the decreasing size of the vesicles since zeta potential was measured on the surface of the outer layer of liposomes. The negative value was contributed by the negatively charged head group of the phosphatidylcholine. All the zeta potential of FL after sonication was recorded with a magnitude higher than $30 \mathrm{mV}$. At the point of 25 minutes with $40 \%$ amplitude, the zeta potential was recorded at $-51.2 \mathrm{mV}$. It was sufficient to maintain a stable system according to the desirable zeta potential (higher than \pm 30 $\mathrm{mV}$ ) remarked by Honary and Zahir (2013). High magnitude of zeta potential exhibited by liposomes led to increased stability of the suspension due to a larger electrostatic repulsion between particles and overcome the natural tendency to aggregate (Ahmed \& Aljaeid, 2016)

Results indicated that these two factors of sonication, which were sonication amplitude and the extent of treatment influenced the size dispersion and zeta potential of liposomes. The sonication set up parameters of $40 \%$ amplitude and treatment time of 25 minutes were selected to prepare loaded liposomes in the following stage. This was attributed to the characteristics of free liposomes at this certain point, which yielded the smallest size of particles with the lowest polydispersity index and a sufficiently high magnitude of zeta potential in accordance with the spectra as shown in Figure 8. It presented that

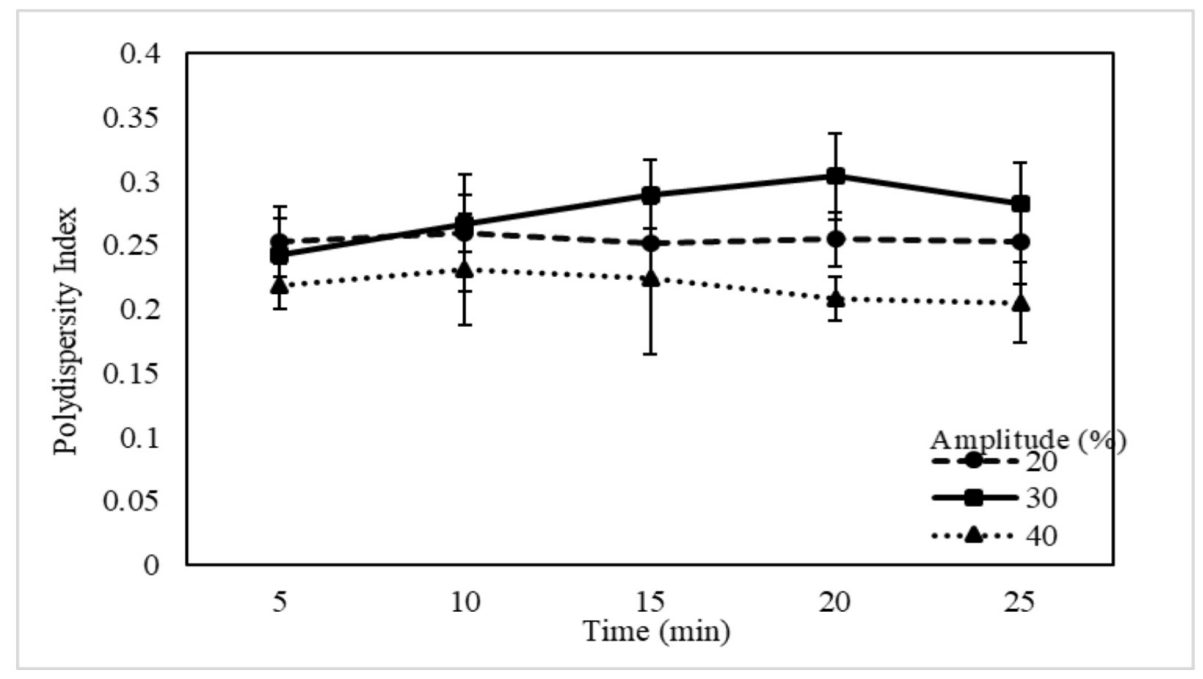

Figure 6: Effect of sonication amplitude and time on polydispersity index of FL 


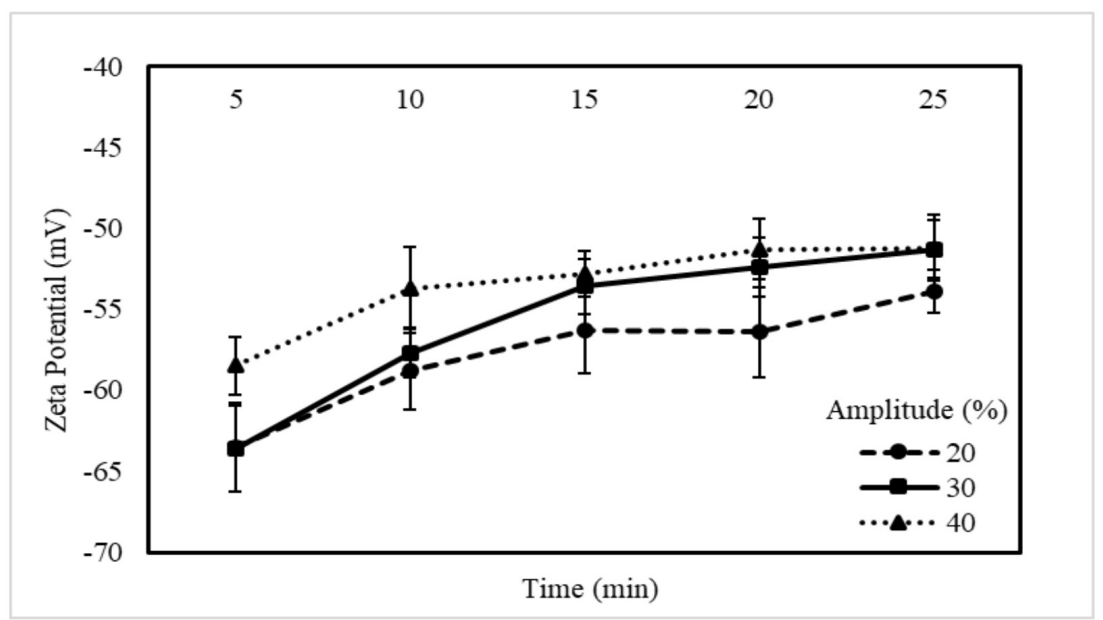

Figure 7: Effect of sonication amplitude and time on zeta potential of FL.

the distribution of FL was uniform, which may be due to a strong mechanical force on liposome through sonication. Subsequently, the vesicle size was greatly reduced and the vesicle was enhanced to be in uniform size distribution. The selected parameters of sonication were regarded as a control set up of liposome preparation before encapsulation of quercetin. The deliberation of these parameters is crucial to attain reliable and repeatable results in synthesizing liposomes.

\section{Effect of Concentration of Quercetin}

The effect of quercetin concentration on the physicochemical characteristics of QL is shown in Figure 9. A gradual increment in particle size $(p<0.05)$ was observed as the concentration of quercetin was increasing. The increasing size of vesicles after drug loading was also reported by

(a)

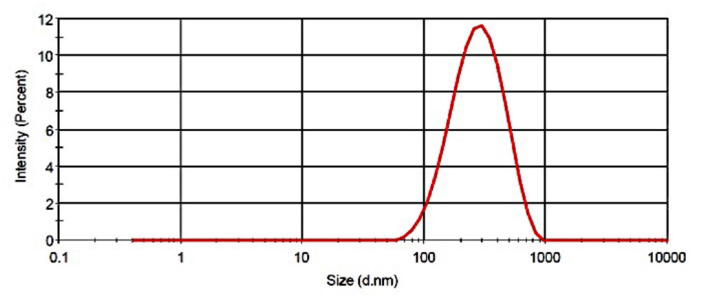

Altin et al. (2018). The expansion of liposome size was attributed to the increasing amount of quercetin entrapped within phospholipid bilayer. Liposomes loaded with quercetin at a higher concentration than $1.5 \mathrm{mg} / \mathrm{ml}$ showed almost a constant value of size. The capacity of liposomes to encapsulate quercetin was determined by adding an extra amount of quercetin in the formulation. Liposomes with quercetin concentration of more than $3.0 \mathrm{mg} / \mathrm{ml}$ were extremely unstable as there were aggregates observed during the storage. It showed that $3.0 \mathrm{mg} / \mathrm{ml}$ was the maximum concentration of quercetin that could be loaded but it unlikely that quercetin could be encapsulated into the liposomes.

In the meantime, the polydispersity indices of the QL for all concentrations were

(b)

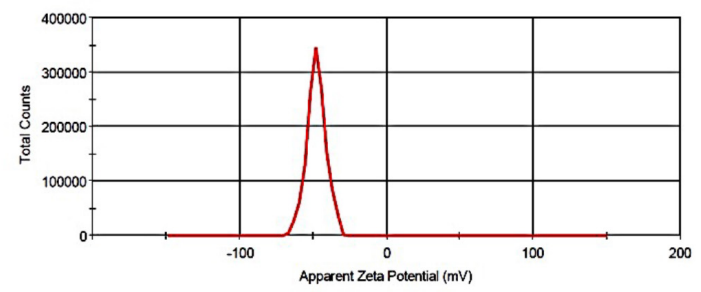

Figure 8: DLS spectra of FL at 40\% amplitude for 25 minutes (a) vesicle size and (b) zeta potential 


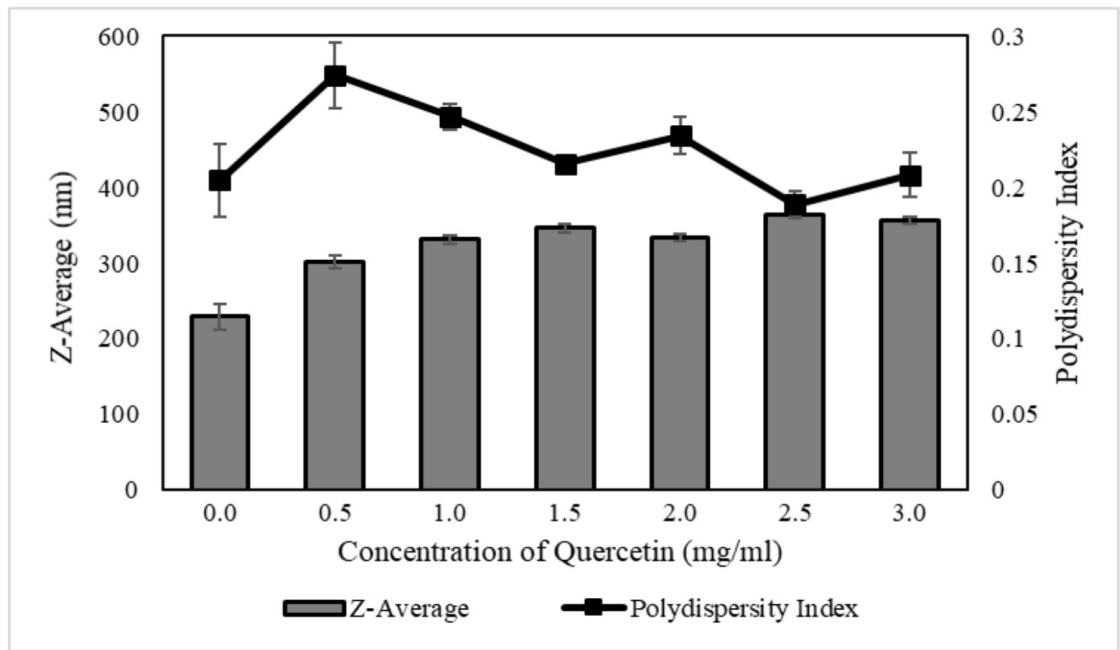

Figure 9: Effect of quercetin concentration on particle size and polydispersity index of QL

varied $(\mathrm{p}<0.05)$ but still lower than 0.3 which means that the vesicles were in uniform size distribution. Meanwhile, the incorporation of neutral-charged quercetin in the liposomes did not remarkably change the zeta potential of the liposomes (result was not shown). The zeta potential of QL of different concentrations were around at the same range as before encapsulation, in betwee -40 to $-50 \mathrm{mV}$, which represents stable suspension was formed. The negative values of zeta potential were due to the composition of the anionic phosphatidylcholine.

The quality of liposome as a nanocarrier is also evaluated by its performance on encapsulation efficiency and drug loading. The solubility of the encapsulated compound in the matrix material will primarily affect the encapsulation efficiency (Kumari et al., 2010). Encapsulation efficiency is the percentage of a bioactive compound that is successfully encapsulated in the liposomes while the loading capacity is the amount of drug loaded per weight of the liposomes. Therefore, these are regarded as the critical factor in inspecting the quality of liposome.

From Figure 10, as the concentration of quercetin increased, the encapsulation efficiency of the liposomes was also increasing $(p<0.05)$ until reaching its peak of $90.7 \%$ at the concentration of $1.5 \mathrm{mg} / \mathrm{ml}$. Meanwhile, the loading capacity of the liposomes was at a plateau after reaching its maximum with 9.4 $\%$ at the similar concentration. At this point, the increase in quercetin concentration most likely did not result in a higher percentage of encapsulation efficiency because the maximum encapsulation ability of the liposome was achieved. Instead, the encapsulation efficiency was slightly reduced after being loaded with quercetin at a concentration of $3.0 \mathrm{mg} / \mathrm{ml}$. This indicates that the remaining amount of quercetin initially added into the formulation could not be entrapped more in the liposomes. Concentration added beyond the liposome ability to encapsulate resulted in free, quercetin molecules which are not encapsulated. Thus, $1.5 \mathrm{mg} / \mathrm{ml}$ is the optimum concentration of quercetin that could be loaded into the liposome to present the highest efficiency as a carrier. This concentration of quercetin was selected to incorporate into other different formulation for further evaluation of the liposomes.

\section{Effect of Formulation of Quercetin-loaded Liposome}

Five formulations of quercetin-loaded liposomes were prepared by varying the composition of phosphatidylcholine and cholesterol to study the effect on the physicochemical characteristics of 


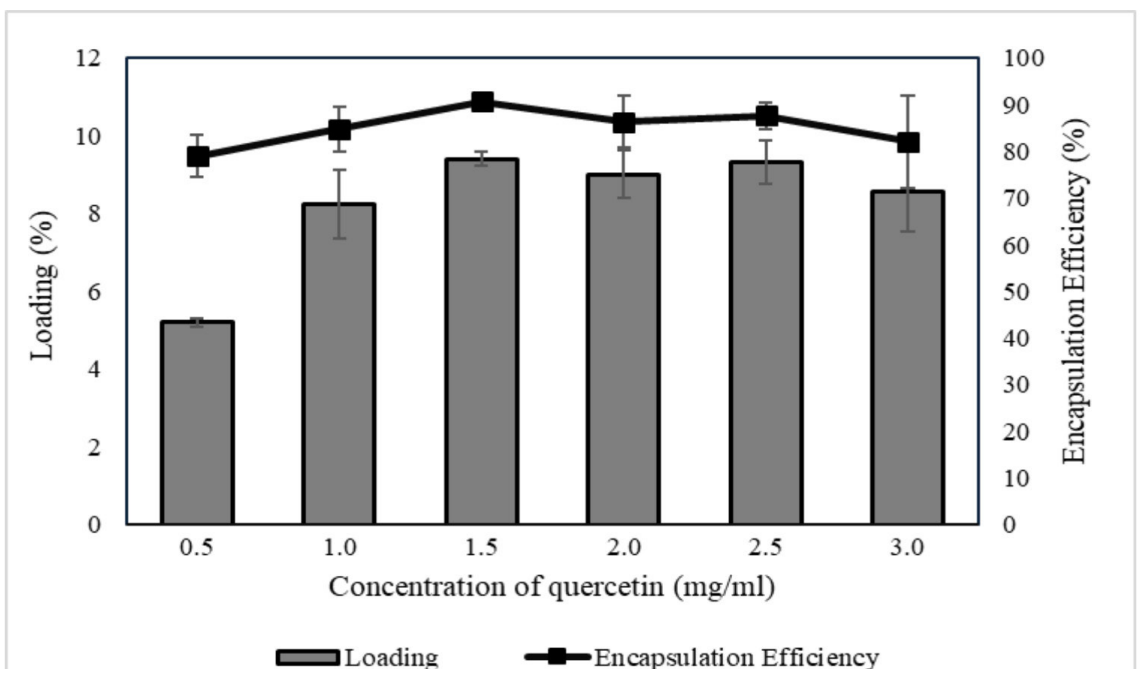

Figure 10: Effect of quercetin concentration on encapsulation efficiency and quercetin loading of QL

QL and its performance on encapsulation. Table 1 presents the data collected from different formulations of QL prepared in triplicate. It shows a significant difference in vesicle size $(\mathrm{p}<0.05)$, which ranges between $289.5 \mathrm{~nm}$ and $406.4 \mathrm{~nm}$ attributed to the different amount of phosphatidylcholine and cholesterol used in the formulation. In terms of PDI and zeta potential, the measurements were differ in relatively small extent $(\mathrm{p}<0.05)$ which depicted a homogeneous and stable liposome suspension that could be prepared following the sonication method. The encapsulation efficiency and quercetin loading also had significant impacts $(\mathrm{p}<0.05)$ due to different formulation of liposome. The subject of its performance on these parameters would be discussed to select the best liposome formulation.

F1 $(8: 0 \mathrm{w} / \mathrm{w})$ yielded the smallest size of liposomes compared to other formulations. However, its efficiency on encapsulation was the lowest among others. Thus, cholesterol was added in other formulations to act as a permeability barrier, which could improve the encapsulation efficiency (Payton et al., 2014). Despite that, excess concentration of cholesterol as in F3 $(8: 2 \mathrm{w} / \mathrm{w})$ had reduced the quercetin loading. The reduction in quercetin loading was also found by Begum et al., (2012) when adding high content of cholesterol in the formulation because there might be a competition between cholesterol and hydrophobic molecules of entrapped material in the limited space of the phospholipid bilayer. Increasing the weight of phosphatidylcholine as in F5 (10:1 w/w) resulted in decreased encapsulation efficiency and quercetin loading. This might be due to the phospholipid membrane being tightly packed

Table 1: Physicochemical characteristics and performance of QL

\begin{tabular}{|c|c|c|c|c|c|}
\hline $\begin{array}{c}\text { Formulation } \\
(\text { w/w) } \\
\text { (SPC:Chol) }\end{array}$ & $\begin{array}{l}\text { Z-Average } \\
\text { (nm) }\end{array}$ & $\begin{array}{l}\text { Polydispersity } \\
\text { Index }\end{array}$ & $\begin{array}{c}\text { Zeta } \\
\text { Potential } \\
(\mathrm{mV})\end{array}$ & $\begin{array}{l}\text { Encapsulation } \\
\text { Efficiency (\%) }\end{array}$ & $\begin{array}{c}\text { Quercetin } \\
\text { Loading (\%) }\end{array}$ \\
\hline F1 (8:0) & $289.5 \pm 4.6$ & $0.20 \pm 0.01$ & $-47.7 \pm 1.4$ & $86.5 \pm 1.8$ & $9.5 \pm 0.5$ \\
\hline F2 (8:1) & $346.4 \pm 5.2$ & $0.22 \pm 0.01$ & $-49.6 \pm 1.2$ & $90.7 \pm 1.2$ & $9.3 \pm 0.3$ \\
\hline F3 (8:2) & $323.4 \pm 5.2$ & $0.26 \pm 0.02$ & $-46.6 \pm 1.5$ & $90.1 \pm 0.6$ & $8.1 \pm 0.1$ \\
\hline F4 (6:1) & $406.4 \pm 4.5$ & $0.22 \pm 0.02$ & $-46.2 \pm 0.5$ & $91.5 \pm 0.6$ & $11.4 \pm 0.4$ \\
\hline F5 (10:1) & $347.1 \pm 8.4$ & $0.20 \pm 0.01$ & $-50.4 \pm 1.3$ & $86.5 \pm 0.7$ & $7.3 \pm 0.4$ \\
\hline
\end{tabular}


with the phosphatidylcholine and cholesterol causing lesser space to incorporate quercetin into the bilayer.

F4 $(6: 1 \mathrm{w} / \mathrm{w})$ seemed to be the best liposome formulation compared to others as it yielded the highest encapsulation efficiency and quercetin loading with $91.5 \%$ and $11.4 \%$ respectively. Reducing the amount of phosphatidylcholine resulted in a higher percentage of quercetin incorporation due to certain liposome loading capacity of lower weight composition. However, while considering the values of average particle size, F2 $(8: 1 \mathrm{w} / \mathrm{w})$ presented more favourable results with a smaller size of liposomes than F4 $(6: 1 \mathrm{w} / \mathrm{w})$ which was $346.4 \mathrm{~nm}$ instead of 406.4 $\mathrm{nm}$. The particle size in F4 (6:1 w/w) was bigger as the phospholipid membrane was loosened due to the lower composition of phosphatidylcholine and the size was getting bigger after quercetin entrapment. The spectra of vesicle size and zeta potential of F2 (8:1w/w) are shown in Figure 11.

F2 (8:1 w/w) also yielded high encapsulation efficiency and quercetin loading which were $90.7 \%$ and $9.3 \%$ respectively. Above all, the best formulation was chosen to be F2 $(8: 1 \mathrm{w} / \mathrm{w})$ because it achieved the desired properties of a nanocarrier with small particle size, low polydispersity index, a high magnitude of zeta potential with a high percentage of encapsulation efficiency and quercetin loading. It was found that varying the lipid formulation produced different properties of liposome and therefore significantly affected its performance.

(a)

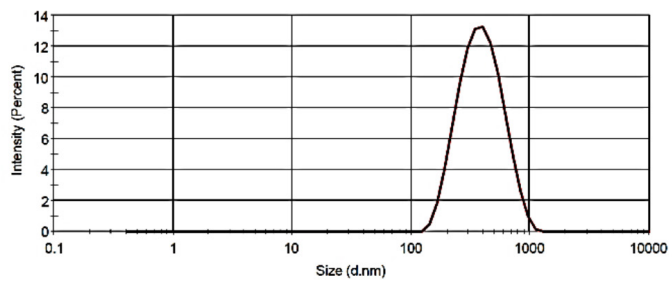

\section{Conclusion}

This present study demonstrated an approach to prepare homogenous QL in aqueous solution applying sonication as a post-treatment method to downsize the vesicle size. Sonication parameters on amplitude and time of treatment were proven to affect the characterization of liposomes in terms of average particle size, polydispersity index and zeta potential. There was also a dependence on phospholipid to cholesterol ratio used in the formulation in preparing liposomes with high entrapping capability. QL had been successfully prepared with high encapsulation efficiency and quercetin loading which were $90.7 \%$ and $9.3 \%$ respectively. The vesicles were spherical and had a narrow size distribution with a high magnitude charge of zeta potential. In conclusion, these features presented by QL synthesized in this study make them appealing and become a promising delivery system not only for quercetin but also can be extended for other drugs or bioactive agents that have diverse therapeutic effects for human health.

\section{Acknowledgements}

The authors would like to express their sincere appreciation to the Ministry of Education, for the grant of the Fundamental Research Grant Scheme (FRGS) and high appreciation to Faculty of Ocean Engineering Technology and Informatics and Central Laboratory, University Malaysia Terengganu for the cooperation and support. This research is part of a dissertation which was submitted as partial fulfilment to meet requirements for the degree of Masters at Universiti Malaysia Terengganu.

\section{(b)}

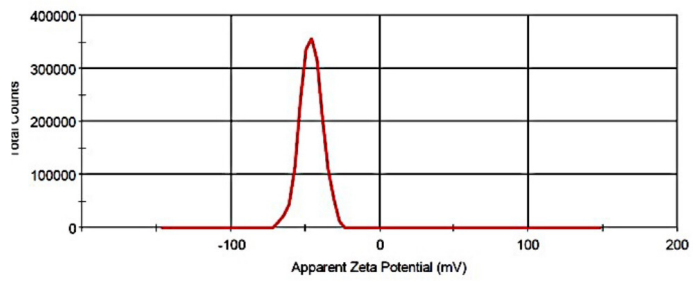

Figure 11: DLS spectraof QL with formulation of 8:1 (w/w) on (a) vesicle size and (b) zeta potential 


\section{References}

Ahmed, T. A., \& Aljaeid, B. M. (2016). Preparation, characterization, and potential application of chitosan, chitosan derivatives, and chitosan metal nanoparticles in pharmaceutical drug delivery. Drug Design, Development and Therapy, 10, 483-507.

Akbarzadeh, A., Rezaei-Sadabady, R., Davaran, S., Joo, S. W., Zarghami, N., Hanifehpour, Y., Samiei, M., Kouhi, M., \& Nejati-Koshki1, K. (2013). Liposome: Classification, preparation, and applications. Nanoscale Research Letters, 8(1), 102-110.

Altin, G., Gültekin-Özgüven, M., \& Ozcelik, B. (2018). Chitosan coated liposome dispersions loaded with cacao hull waste extract: Effect of spray drying on physicochemical stability and in vitro bioaccessibility. Journal of Food Engineering, 223, 91-98.

Anwekar, H., Patel, S., \& Singhai, A. K. (2011). Liposome-As drug carriers. International Journal of Pharmacy \& Life Sciences, 2(7), 945-951.

Barba, A. A., Bochicchio, S., Lamberti, G., \& Dalmoro, A. (2014). Ultrasonic energy in liposome production: Process modelling and size calculation. Soft Matter, 10, 25742581 .

Begum, M. Y., Abbulu, K., Sudhakar, M., \& Anees, A. (2012). Celecoxib-encapsulated liposomes of long alkyl chain lipids: Formulation, characterization and in vitro performance. Der Pharmacia Sinica, 3(1), 117-125.

Bozzuto, G., \& Molinari, A. (2015). Liposomes as nanomedical devices. International Journal of Nanomedicine, 10, 975-999.

Bulbake, U., Doppalapudi, S., Kommineni, N., \& Khan, W. (2017). Liposomal formulations in clinical use: An updated review. Pharmaceutics, 9(2), 12-45.

Çağdaş, M., Sezer, A. D., \& Bucak, S. (2014). Liposomes as potential drug carrier systems for drug delivery. In Application of Nanotechnology in Drug Delivery. IntechOpen.

Dag, D., \& Oztop, M. H. (2017). Formation and characterization of green tea extract loaded liposomes. Journal of Food Science, 82(2), 463-470.

David, A. V. A., Arulmoli, R., \& Parasuraman, S. (2016). Overviews of biological importance of quercetin: A bioactive flavonoid. Pharmacognosy Reviews, 10(20), 84-89.

Elmeshad, A. N., Mortazavi, S. M., \& Mozafari, M. R. (2014). Formulation and characterization of nanoliposomal 5-fluorouracil for cancer nanotherapy. Journal of Liposome Research, 24(1), 1-9.

Hao, J., Guo, B., Yu, S., Zhang, W., Zhang, D., Wang, J., \& Wang, Y. (2017). Encapsulation of the flavonoid quercetin with chitosancoated nano-liposomes. LWT-Food Science and Technology, 85, 37-44.

He, H., Lu, Y., Qi, J., Zhu, Q., Chen, Z., \& Wu, W. (2018). Adapting liposomes for oral drug delivery. Acta Pharmaceutica Sinica $B, 9(1), 36-48$.

Honary, S., \& Zahir, F. (2013). Effect of zeta potential on the properties of nano-drug delivery systems-A review (Part 2). Tropical Journal of Pharmaceutic Al Research, 12(2), 265-273.

Kalepu, S., Sunilkumar, K. T., Betha, S., \& Mohanvarma, M. (2013). Liposomal drug delivery system-A comprehensive review. International Journal of Drug Development and Research, 5(4), 62-75.

Karami, N., Moghimipour, E., \& Salimi, A. (2018). Liposomes as a novel drug delivery system: Fundamental and pharmaceutical application. Asian Journal of Pharmaceutics, 12(1), 31-41.

Kumar, S., \& Pandey, A. K. (2013). Chemistry and biological activities of flavonoids: An overview. The Scientific World Journal, 2013, 1-16. 
Kumar, V. D., Verma, P. R. P., \& Singh, S. K. (2015). Development and evaluation of biodegradable polymeric nanoparticles for the effective delivery of quercetin using a quality by design approach. LWT-Food Science and Technology, 61(2), 330-338.

Kumari, A., Yadav, S. K., Pakade, Y. B., Singh, B., \& Yadav, S. C. (2010). Development of biodegradable nanoparticles for delivery of quercetin. Colloids and Surfaces B: Biointerfaces, 80(2), 184-192.

Laouini, A., Maalej, C. J., Blouza, I. L., Sfar, S., Charcosset, C., \& Fessi, H. (2012). Preparation, characterization and applications of liposomes: State of the art. Journal of Colloid Science and Biotechnology, 1(2), 147-168.

Li, Z., Liu, M., Wang, H., \& Du, S. (2016). Increased cutaneous wound healing effect of biodegradable liposomes containing madecassoside: Preparation optimization, in vitro dermal permeation, and in vivo bioevaluation. International Journal of Nanomedicine, 11, 2995-3007.

Naeem, S., Kiew, L. V., Chung, L. Y., Ashraf, M. A., \& Misran, M. (2016). Liposomes as amphiphilic carriers: Encapsulation and stability aspects. Sains Malaysiana, 45(1), 71-77.

Nam, J. S., Sharma, A. R., Nguyen, L. T., Chakraborty, C., Sharma, G., \& Lee, S. S. (2016). Application of bioactive quercetin in oncotherapy: From nutrition to nanomedicine. Molecules, 21(1), 108.

Rezaei-Sadabady, R., Eidi, A., Zarghami, N., \& Barzegar, A. (2016). Intracellular ROS protection efficiency and free radical-scavenging activity of quercetin and quercetin-encapsulated liposomes. Artificial Cells, Nanomedicine, and Biotechnology, 44(1), 128-134.

Panche, A. N., Diwan, A. D., \& Chandra, S. R. (2016). Flavonoids: An overview. Journal of Nutritional Science, 5(47), 1-15.

Payton, N. M., Wempe, M. F., Xu, Y., \& Anchordoquy, T. J. (2014). Longterm storage of lyophilized liposomal formulations. Journal of Pharmaceutical Sciences, 103(12), 3869-3878.

Silva, R., Ferreira, H., Little, C., \& Paulo, A. C. (2010). Effect of ultrasound parameters for unilamellar liposome preparation. Ultrasonics Sonochemistry, 17(3), 628632.

Tefas, L. R., Muntean, D. M., Vlase, L., Porfire, A. S., Achim, M., \& Tomuță, I. (2015). Quercetin-loaded liposomes: Formulation optimization through a D-optimal experimental design. Farmacia, 63(1), 2633.

Wu, C. H., Shieh, T. M., Wang, K. L., Huang, T. C., \& Hsia, S. M. (2015). Quercetin, a main flavonoid in onion, inhibits the PGF $2 \alpha$ induced uterine contraction in vitro and in vivo. Journal of Functional Foods, 19, 495-504.

Zhao, L., \& Temelli, F. (2017). Preparation of anthocyanin-loaded liposomes using an improved supercritical carbon dioxide method. Innovative Food Science and Emerging Technologies, 39, 119-128.

Zidan, A. S., \& Aldawsari, H. (2015). Ultrasound effects on brain-targeting mannosylated liposomes: In vitro and blood-brain barrier transport investigations. Drug Design, Development and Therapy, 9, 3885-3898. 